\title{
Mechtild Düsing, Rechtsanwältin und Notarin, Münster
}

\author{
Juristinnen machen Karriere - wir stellen sie vor
}

\begin{abstract}
Das Interview führte Anke Gimbal, Geschäftsführerin, Deutscher Juristinnenbund e.V., am 20. März 2014 in Berlin.
\end{abstract}
\begin{abstract}
Was hat Dich bewegt, nach dem Abitur Jura zu studieren? Lehrerin wollte ich nicht werden. Ich wollte etwas Schönes studieren und es fielen mir Kunstgeschichte und Archäologie ein, die mich interessierten. Zu dem Zeitpunkt hatte ich noch kein Vorbild, was Jura anbelangt. Die Kanzlei meines Onkels, der Rechtsanwalt war, hatte ich, als ich nach der Schule mit dem Studium anfing, noch nie gesehen. Ich fand Kunstgeschichte und Archäologie auch hochinteressant und war eine erfolgreiche Studentin. Aber was sollte ich mit Kunstgeschichte und Archäologie werden: Museumsleiter waren damals nur Männer und Archäologie war mir zu exotisch, um daraus einen Beruf zu machen. Ich hatte gar kein richtiges Berufsbild dazu. Meine Eltern haben mir dann empfohlen, Jura auszuprobieren. Das tat ich, fand es spannend und studierte alles mehrere Semester nebeneinander. Da mir Jura flexibler erschien, denn als Juristin kann man an jeder Stelle in Deutschland sein Büro aufmachen, habe ich mich später alleine auf Jura konzentriert.
\end{abstract}

\section{Was hat Dich nach Münster verschlagen?}

Nach Münster hat mich die Liebe verschlagen. Ich studierte in München, hatte aber einen Freund aus Münster, der unbedingt wollte, dass ich dorthin komme. Das habe ich gemacht, sonst wäre ich nun höchstwahrscheinlich Rechtsanwältin in München. Aber kaum war ich in Münster, ging es mit der Liebe auseinander. Dann war ich wieder neu verliebt und bin in Münster geblieben. Wahrscheinlich war es gut, dass ich nach Münster gegangen bin, da ich Westfälin bin. Ich komme aus Gelsenkirchen und es hat mir in Münster auch gut gefallen.

Wie hast Du das Studium in den 6oer Jahren - der Kinder, Küche, Kirche-Zeit - erlebt?

Ich habe nie gesagt „Kinder, Küche, Kirche“, hatte aber auch drei Ks, und zwar „Kanzlei, Kinder, Kommunalpolitik“. 1964 habe ich Abitur gemacht. Meine Mutter war Hausfrau und Mutter. Das war das abschreckende Beispiel schlechthin. Auf keinen Fall wollte ich Hausfrau und Mutter werden, das wusste ich schon als Kind. Diese Abhängigkeit von einem Mann, sei er noch so nett, geht gar nicht. Ich habe gesagt, Studium und Kanzlei sind erst einmal wichtig. Auch Kinder habe ich erst nicht wahrgenommen; sie kommen dann oder sie kommen nicht. Als ich zwischen 39 und 41 Jahre alt war, kamen sie allerdings und das war auch sehr schön. Meine Kinder habe ich als Glück empfunden, aber mein Hauptziel waren Selbstständigkeit und Unabhängigkeit.

Während meines Studiums habe ich keine Diskriminierungen erlebt. Ich war eine sehr gute Studentin, sah gut aus und wurde von den Professoren eher hofiert. Ich erinnere mich an eine Arbeitsrechtsvorlesung, bei der Klausuren zurück gegeben wurden. Ich wollte anschließend ins Theater gehen und hatte ein ganz
Mechtild Düsing, geb. 1944 in Bilstein Lennestadt, studierte von 1965 bis 1969 Rechtswissenschaft an den Universitäten Münster und München. Nach ihrem Referendariat beim OLG Hamm und dem Abschluss des Zweiten Staatsexamens 1973 in Düsseldorf war sie zunächst als Rechtsanwältin in einer Einzelpraxis tätig und gründete 1975 mit Kollegen die Kanzlei Meisterernst Düsing Manstetten in Münster. 1983 erfolgte ihre Ernennung zur Notarin, 1988 wurde sie zunächst Fachanwältin für Verwaltungsrecht mit Schwerpunkt Studienplatzklagen. 2006 wurde sie auch Fachanwältin für Erbrecht.

Zwischen 1990 und 2006 vertrat sie insbesondere Landwirte vor den Europäischen Gerichten in Luxemburg in Schadensersatzprozessen gegen die Europäische Gemeinschaft. Sie ist seit 2009 Vorsitzende der Arbeitsgemeinschaft Agrarrecht im Deutschen Anwaltverein (DAV). Seit 2009 ist sie Vorsitzende des Fachanwaltsausschuss Agrarrecht der Rechtsanwaltskammer Hamm, seit 2010 Fachanwältin für Agrarrecht. Sie veröffentlichte zahlreiche Publikationen und Vorträge zum Landwirtschaftsrecht, Anwaltsrecht und zu Frauen in der Anwaltschaft.

Mechtild Düsing ist seit 2001 Mitglied des Verfassungsrechtsausschusses des DAV. Seit 2011 ist sie Mitglied des Vorstands des DAV. Von 2004 bis 2009 war sie Vorsitzende der Arbeitsgemeinschaft Anwältinnen im DAV und ist seitdem Vorstandsmitglied. Sie ist Genderbeauftragte des DAV-Vorstandes und Vorsitzende des Genderausschusses des DAV. Außerdem ist sie Mitglied der Arbeitsgemeinschaften Erbrecht, Internationaler Rechtsverkehr und Anwaltsnotariat im DAV.

Weiterhin ist sie Mitglied der European Women Lawyers Association (EWLA), von ZONTA International, dem Westfälischen Kunstverein Münster (Mitglied des Beirats), der Bürgerstiftung Münster (Mitglied im Kuratorium) und der Atlantik-Brücke e.V. Berlin.

Mechtild Düsing ist seit 1981 Mitglied des djb und seit 1994 Vorstandsmitglied (Schatzmeisterin) der Regionalgruppe Münster. Sie engagiert sich in zahlreichen djb-Projekten, u.a. initiierte sie 2009 die Aktion „Aktionärinnen fordern Gleichberechtigung - Erhöhung des Frauenanteils in Führungspositionen deutscher Unternehmen“.

Sie ist verheiratet und hat drei Kinder.

schickes lila Seidenkleid an, mit dem ich mich in der Klausurbesprechung fehl am Platze fühlte. Als der Professor sagte, die beste Klausur habe eine Dame geschrieben, guckten mich alle an und ich brach in Schweiß aus. Das war unangenehm. Aber sonst hatte ich keine Probleme. Die kamen erst später, als ich Anwältin war. Ich habe in den ersten eineinhalb Jahren angefangen mit einer kleinen Kanzlei in meiner Wohnung. Dann klingelten die Mandanten bei Frau Rechtsanwältin Düsing und ich machte die Tür auf, weil ich ja noch keine Angestellten hatte. Sie hielten mich für die Sekretärin und sagten, ich möchte zu Frau Rechtsanwältin 
Düsing. In der Situation bekam ich auch leicht Schweißausbrüche, denn ich musste den Mandanten davon überzeugen, dass er trotzdem richtig ist. Nach einem halben Jahr hatte ich einen Lehrling, eine nette junge Frau, die später Rechtspflegerin wurde und die die Tür aufmachte. Das war schon besser.

Ein Erlebnis möchte ich schildern. Meine Mutter besuchte mich in München und ich zeigte ihr das juristische Seminar. Es war gerade Vorlesungsende. Über eine große Treppe kamen alle Studenten herunter und ich stand mit meiner Mutter unten. Sie blickte die Treppe hinauf und sagte ganz entsetzt, „mein Gott Kind, so viele Männer“. Bis zu dem Zeitpunkt war mir das noch gar nicht so aufgefallen. Erst als sie das sagte, dachte ich, das stimmt, nur Männer. Es gab so gut wie keine Studentinnen.

\section{Hattest Du familiären Rückhalt bei deinen Zukunftsplänen? \\ Meine Eltern haben mir alle Freiheit gelassen und mir alles fi- nanziert. Sie haben meine Zukunftspläne voll unterstützt. Sobald ich 1969 das Erste Staatsexamen hatte, war ich selbstständig und habe kein Geld mehr von meinen Eltern genommen. Ich habe gemacht, was ich wollte, und habe meinen Eltern nur irgendwann einmal mitgeteilt, ich heirate jetzt.}

\section{Hattest Du Vorbilder? Und warst Du immer so selbstsicher wie heute?}

Vorbild war mein Onkel, ein Rechtsanwalt in Bonn. Ich habe mir die Kanzlei angesehen und auch mit ihm gesprochen. Er verteidigte damals einen Mitarbeiter des für den Contergan-Skandal verantwortlichen Pharmaunternehmens. Ein positives Vorbild einer Frau hatte ich nicht. Meine Vorbilder waren nur Männer. Und meine Mutter war das, wovon ich mich absetzen wollte, das war mir ganz, ganz wichtig. Im Nachhinein stelle ich aber fest, dass ich ganz viel genauso gemacht habe wie meine Eltern. Ich bin selbstständig wie mein Vater, der selbstständiger Kaufmann war. Ich habe ein sehr schönes Haus mit großem Garten, das hatten wir auch zu Hause. Ich habe drei Kinder wie meine Eltern. Genauso ein schönes Auto, das hatten meine Eltern auch immer - übrigens auch meine Mutter. Wenn ich das so anschaue, läuft ganz viel gleich. Einzig wurde ich nicht Hausfrau, sondern ich habe die Rolle des Vaters übernommen und auch die ganze Familie ernährt.

\section{Wolltest Du Rechtsanwältin und/oder Notarin werden und hattest Du deine Karriere schon während des Studiums geplant oder gab es ein anderes Ziel?}

Ich wollte von Anfang an Rechtsanwältin werden. Zumindest nachdem ich mich für Jura entschieden hatte, war Rechtsanwältin sozusagen mein Examensziel. Selbstständigkeit fand ich attraktiv und außerdem dachte ich, als selbstständige Anwältin ist man flexibler, auch im Hinblick auf die Gründung einer Familie. Mir schien es besser eine Anwältin zu sein, die selbst entscheiden kann, wo sie arbeiten will und was sie arbeiten will. Ich hatte die Gelegenheit, nach dem Zweiten Staatsexamen in der Staatskanzlei in NRW anfangen zu können. Sie wollten mich werben und hatten mich eingeladen zum Gespräch mit dem Staatssekretär. Sie hatten mir in schillernden Farben geschildert, was ich da werden könnte. Aber ich habe dankend abgelehnt.

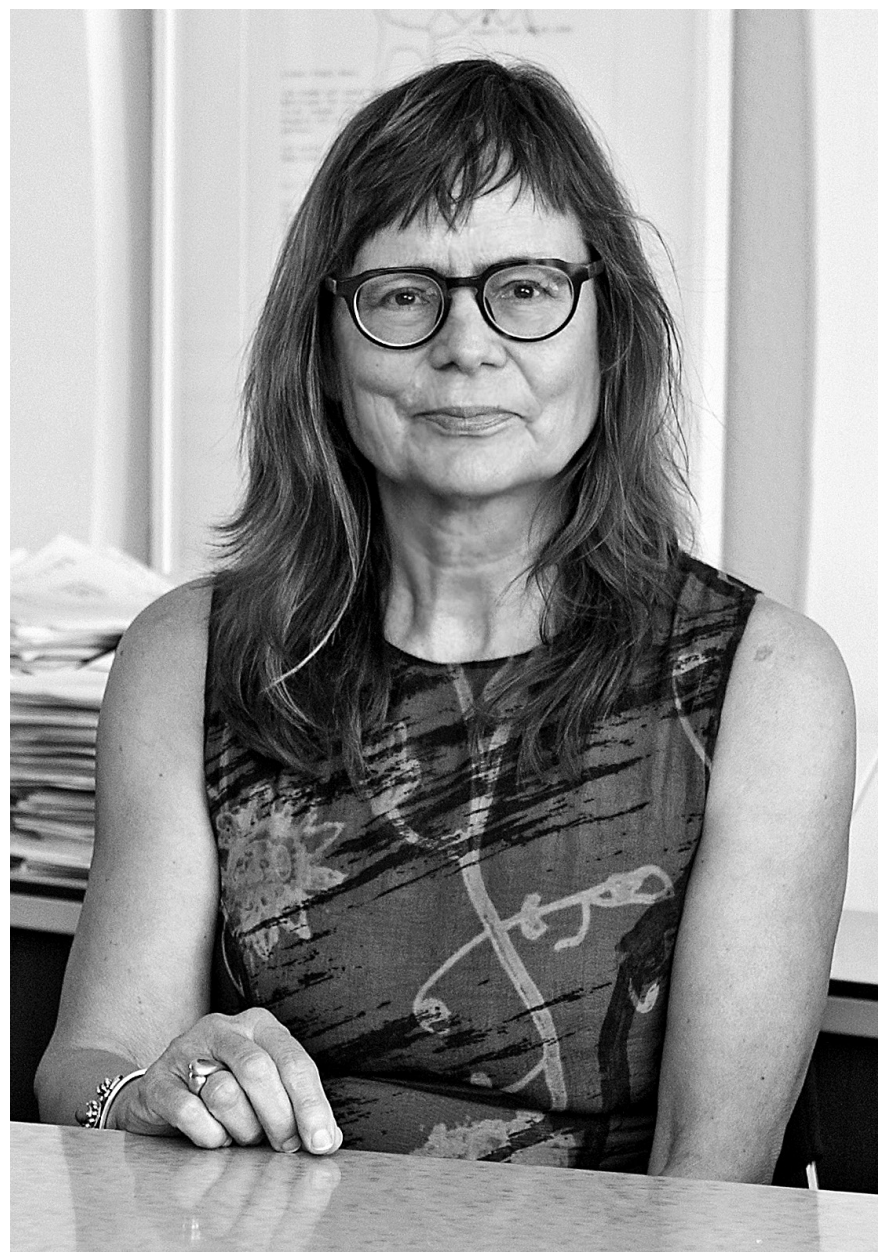

A Mechtild Düsing, Rechtsanwältin und Notarin (Foto: Ralf Emmerich)

Wie bist Du zur Landwirtschaft bzw. zum Agrarrecht und dann auch noch auf europäischer Ebene gekommen?

Ich habe mir nicht vorgenommen, ich werde jetzt Anwältin und arbeite auf dem und dem Rechtsgebiet. Das wird heute oft so gemacht, und das kann man ja auch, wenn man es richtig plant. Ich habe das mehr dem Zufall überlassen und genommen, was sich bot. Von einem Kollegen habe ich die Numerus ClaususZulassungsverfahren geerbt. Er hatte in seiner Kanzlei Numerus Clausus-Prozesse und gab mir welche ab; das war zunächst meine große Geldquelle. Es war eine tolle Arbeit, interessant und brachte auch Geld. Man kam in der ganzen Bundesrepublik herum und lernte nette Kollegen kennen, mit denen man zusammenarbeiten konnte. Das habe ich einfach immer weiter gemacht.

Auch zum Agrarrecht kam ich rein zufällig. Otto Schily, den ich aus Berlin kannte, rief mich an und sagte, hier sind alternative Bauern aus dem Münsterland, die etwas über die Milchquote wissen wollen. Also habe ich mich der Milchquote und dem Agrarrecht angenommen. Über kurz oder lang war ich dann die Milchquoten-Expertin für ganz Deutschland, es kamen die Bauern von Bayern bis Schleswig-Holstein zu mir. Das war eine ganz spannende Geschichte und hat auch Spaß gemacht juristisch und wegen der Mandanten interessant. Man kam in ganz Deutschland herum und es ging bis zum Europäischen Gerichtshof. Bauern sind interessante Mandanten, die auch immer bezahlen. Daran muss man schließlich auch denken. 


\section{Gab es andere Kanzleien in Deutschland, die sich dem Thema Milchquote gewidmet haben?}

Es gab schon noch Kanzleien, die sich auch damit beschäftigt haben, aber wenige und nicht so intensiv wie ich. Es ging los 1984 mit Einführung der Milchquote. 1984 kam der Anruf von Otto Schily und in dem Jahr wurde auch mein Sohn Gustav geboren, daran mache ich das immer fest. Es ging 20 Jahre, ungefähr bis Mitte 2000 .

\section{Bist Du inzwischen Europäerin?}

Das war alles europäisches Recht, womit ich mich unheimlich gerne befasst habe. Ich habe das europäische Recht verstehen gelernt, mit der Kommission verhandelt, vor Gericht gestritten und habe den Eindruck gewonnen, dass Europa auch wirklich gut funktioniert. Man konnte als kleine Anwältin aus Münster einen Bauern vertreten, bis zum Europäischen Gerichtshof gehen und dabei auch noch Recht kriegen. Manchmal habe ich die Mandanten nach Luxemburg mitgenommen. Die waren dann total beeindruckt von dem ganzen Apparat und von den Übersetzern. Ich bin überzeugt von Europa und ich bin der Meinung, dass Europa eine gute Sache ist und auch fortentwickelt werden muss.

\section{Was war Dein größter beruflicher Erfolg?}

Das ist schwer zu sagen. Ich bin jetzt 40 Jahre Anwältin. Die Milchquoteprozesse beim Europäischen Gerichtshof waren immer eine tolle Sache, die mir großen Spaß gemacht hat. Die meisten Prozesse habe ich auch gewonnen. Die Verhandlungen in Brüssel und in Luxemburg vor dem Gerichtshof waren insgesamt eine sehr spannende Zeit. Wir haben auch mit Anwälten aus Belgien, Irland, England und den Niederlanden zusammen gearbeitet. Wir trafen uns in einer Brüsseler Kanzlei und besprachen die Taktik und das weitere Vorgehen. Beruflich war es die interessanteste Zeit und es waren auch die schönsten Erfolge.

\section{Wie hat es geklappt mit Kindern und Karriere?}

Fulltime-Kanzlei und gleichzeitig drei Kinder großziehen, die ich innerhalb von vier Jahren bekommen habe, war anstrengend. Zumal ich keinen Mann an der Seite hatte, der mitgemacht hätte, denn er war ständig auf einer Facharztausbildung und nicht zu Hause. Es lag alles auf meinen Schultern. Als er dann endlich den Facharzt hatte, ging die Ehe in die Brüche und ich war ganz alleine mit den Kindern. Er hat die Kinder höchstens alle 14 Tage zum Wochenende geholt und viel Kindesunterhalt hat er auch nicht gezahlt. Das wünsche ich keinem. Der Jüngste studiert noch und macht seinen Master in Geowissenschaft in Potsdam. Meine Tochter macht gerade Examen in Medizin. Deswegen arbeite ich noch, denn sonst könnte ich das nicht bezahlen.

Solange die Kinder klein waren, habe ich von montags bis freitags acht Stunden täglich gearbeitet und die Wochenenden frei genommen. Morgens habe ich die Kinder in den Kindergarten gebracht oder bin zusammen mit ihnen mit dem Fahrrad zur Schule gefahren, von da aus zur Kanzlei. Mittags bin ich nach Hause gekommen und habe mich mit den Kindern zusammen an den gedeckten Mittagstisch gesetzt. Abends bin ich viertel nach sechs zu Hause gewesen, denn dann ging die Haushälterin. Wenn die Haushälterin krank war, habe ich einen Lehrling aus dem Büro zum Aufpassen nach Hause geschickt. Damals war ich noch in der Kommunalpolitik tätig. Einmal im Monat war Bezirksparlamentssitzung, da war ich nicht zu Hause, und Fraktionssitzung, da kamen die Genossen zu mir. Bezirksbürgermeisterin, Kanzlei, drei Kinder ließen sich so noch vereinbaren. Aber es ging nur mit einer Hauswirtschafterin und einem Hauswirtschaftslehrling, also einem richtigen Betrieb zu Hause. Damals gab es überhaupt keine Kitas und keine Ganztagsschulen. Kindergärten gingen bis zwölf Uhr mittags und dann, wenn man wollte, noch einmal von zwei bis vier. Deswegen musste man Hauswirtschaftspersonal haben. Dafür ging viel Geld drauf, aber das habe ich gerne bezahlt. $\mathrm{Zu}$ Hause bleiben kam für mich nicht in Frage.

Die Entscheidung habe ich natürlich nicht bereut. Ich habe unheimlich gerne Kinder bekommen. Ich fand das toll. Aber wenn ich vor die Alternative gestellt worden wäre, Kinder oder Beruf, hätte ich mich wohl für den Beruf entschieden. Glücklicherweise wurde ich vor diese Entscheidung nicht gestellt und da niemand anderes da war, der das Geld verdient hätte, stellte sich die Frage ohnehin nicht.

\section{Was empfiehlst Du Kolleginnen, wie sie Familie und Arbeit} unter einen Hut bekommen?

Man muss Prioritäten setzen und sich auf jeden Fall die Hausarbeit vom Hals halten. Ich habe mich am Wochenende und abends um die Kinder gekümmert und habe dann auch gekocht. Die Zutaten für das Essen, das ich kochen wollte, habe ich mir vorher einkaufen lassen und konnte dann am Wochenende wunderbar Hausfrau spielen. Heute mit Kinderkrippe und Ganztagsschule müsste es eigentlich gehen. Man muss nicht mehr so viel Geld aufwenden, um sich freizukaufen, wie ich das damals musste. Aber Geld muss man in die Hand nehmen, sonst funktioniert es nicht. Vom Einkommen, das man als Anwältin hat, muss man einen Teil dafür ausgeben, sonst hat man zu wenig Zeit, sich um den Beruf zu kümmern. Man muss nicht gute Hausfrau und gute Köchin sein und gleichzeitig gute Anwältin und gleichzeitig gute Mutter. Alles gleichzeitig geht nicht, das ist ganz klar.

Die Kinder haben sich nicht beschwert. Am Anfang wussten sie es nicht, dann haben sie bemerkt, dass andere Mütter ganz anders ticken. Natürlich haben sie auch mitgekriegt, dass alles auf meinen Schultern lastete und ich das Geld für die ganze Familie verdienen musste. Außerdem hatte ich immer nette Leute im Haushalt - eine wunderbare Haushälterin und als sie kündigte habe ich den Lehrling, einen Mann, als Hauswirtschafter eingestellt. Das fanden die Kinder auch ganz schön und er war bei uns, bis mein jüngster Sohn das Abitur hatte. Jetzt habe ich eine Haushälterin, die nur noch vier Stunden am Tag kommt. Und ich kaufe immer noch nicht selbst ein.

\section{Wie erlebst Du das Berufsleben heute im Vergleich zu früher? Ist Gleichberechtigung noch ein Thema? Gibt es Unterschiede bei der Anwaltschaft und im Notariat?}

Als ich 1973 als Anwältin anfing, war ich exotisch. Es gab noch die Sammeltermine bei den Landgerichten - 20 Sachen gleichzeitig um 9.00 Uhr, wo nur Anträge gestellt wurden. Da kam ich 
rein als junge Anwältin mit Minirock, der ganze Saal voll von diesen schwarzen Krähen und alle drehten sich nach mir um. Das war schon furchtbar und ich bin jedes Mal ins Schwitzen geraten, weil ich das so unangenehm fand. Aber heute ist man als Frau nicht mehr alleine und es gibt diese Sammeltermine nicht mehr. Die Richter waren immer ganz nett, aber mit den Kollegen gab es doch öfter Probleme, denn sie versuchten, mich von oben herab zu behandeln und meinten, mit der Kleinen werden wir schon fertig. Ich hatte oft das Gefühl, nicht so gleichberechtigt behandelt bzw. nicht als vollwertiger Gegner angesehen zu werden.

\section{Worauf sollten die jungen Kolleginnen bei ihrer Karriereplanung achten? Was ist wichtig, wo liegen die Stolpersteine für Frauen? Manchen Kolleginnen fehlt der Ehrgeiz, etwas selber aufbauen zu wollen. Den muss man haben, sonst wird das nichts. Und man darf nicht die Mutterrolle in dem Maße ausfüllen wollen, wie sie einem immer noch von der Gesellschaft nahe gebracht wird. Junge Kolleginnen, die ein Jahr mit Elterngeld zu Hause bleiben wollen, verstehe ich nicht. Für die Kanzlei ist das schädlich. Man kann sich das Baby zum Stillen bringen lassen - das habe ich alles so gemacht und es war kein Problem. Wenn man vom Gehalt etwas für die Kinderbetreuung abgibt, wird es einem hundert Mal wieder zurückgezahlt. Man hat eine gut gehende Kanzlei, die Mandanten bleiben treu. Das lohnt sich in jedem Fall. Sonst sind nach einem Jahr die Mandanten weg.}

\section{Warum bist Du djb-Mitglied geworden und engagierst Dich in zahlreichen djb-Projekten?}

Der djb ist eine tolle Vereinigung emanzipierter Frauen. Das hat mich immer fasziniert, auch weil da Juristinnen aus allen Berufen sind. Deswegen engagiere ich mich. Ich weiß nicht mehr, wann ich den djb kennen gelernt habe, aber ich dachte, da muss ich mitmachen. Bei djb-Veranstaltungen fühle ich mich wohl und versuche, immer mindestens einen Tag zu kommen, weil es einfach schön ist, mit so vielen intelligenten Frauen zusammenzusitzen, zu sprechen und zu lernen. Ich finde die Arbeit unterstützenswürdig. Daher bin ich seit 20 Jahren Schatzmeisterin der Regionalgruppe Münster. Ich organisiere jedes Jahr den Sommerempfang der Regionalgruppe in meinem Garten - die Nachfolgeveranstaltung von Ursula Nelles Spargelessen.

\section{Du engagierst Dich umfangreich ehrenamtlich, nicht nur im} djb, sondern auch in der Kommunalpolitik und im DAV?

Kommunalpolitik mache ich nicht mehr. Ich wollte damals in den Rat der Stadt Münster, wurde von der SPD aufgestellt und habe auch Wahlkampf gemacht. Aber sie haben mich mit einem sehr schlechten Listenplatz versehen, denn denen war ich zu emanzipiert oder vielleicht auch zu elitär. Mein Wahlkreis war der, in dem ich wohne. Es ist eine sehr schöne Gegend, aber das hat die Folge, dass die CDU dort die Mehrheit hat. Den Wahlkreis konnte ich also nicht gewinnen und hätte einen besseren Listenplatz haben müssen. Daraufhin habe ich Schluss mit der Kommunalpolitik gemacht und beschlossen, mich beim DAV zu engagieren.

\section{Werden die Interessen der Anwältinnen im DAV ausreichend berücksichtigt?}

Im DAV-Vorstand mache ich Anwaltspolitik und auch sehr viel Anwältinnenpolitik. Ich hatte mir vorgenommen, die Anwältinnen im DAV vorwärts zu bringen und trete dem Vorstand gendermäßig auf die Füße. Auch bei der Vorstandssitzung gestern und heute gab es z.B. kontroverse Diskussionen bei der Frage, wie viele Anwältinnen bzw. Referentinnen bei den Veranstaltungen des DAV und der Anwaltsakademie dabei sind. Teilweise gibt es Veranstaltungen ganz ohne Frauen, mal sind es zehn Männer und eine Frau bei den Rednern. So geht es nicht weiter. Es sollte eine Resolution verabschiedet werden, wo der Vorstand sich verpflichtet, mindestens ein Drittel Frauen als Rednerinnen vorzusehen. Die Anwaltsakademie und die Arbeitsgemeinschaften haben sich dem widersetzt, so dass die Resolution nur sehr abgeschwächt verabschiedet wurde. Die Arbeitsgemeinschaften werden nun aufgefordert auf eine Podienbesetzung mit einem Drittel Referentinnen hinzuwirken. Wenn das nicht gelingt, soll es begründet werden. Fast auf jeder zweiten Vorstandssitzung gibt es ein solches Genderthema, denn das Thema Gleichstellung ist im DAV noch nicht erledigt. In den Gremien des DAV, gerade auch in den entscheidenden Positionen, sind immer noch nicht genug Frauen. Es gibt in den ca. 30 Ausschüssen, die den Vorstand bei Gesetzgebungsverfahren beraten, nur ganz wenige Vorsitzende Frauen. Auch weibliche Mitglieder sind in einer erheblichen Minderzahl. Eine Präsidentin hatte der DAV - wie auch die BRAK - noch nie. Mein Ziel ist; der nächste Präsident wird eine Präsidentin.

\section{Wie bist Du auf den Gedanken gekommen, zur Hauptversammlung eines DAX-Konzerns zu gehen und Fragen an Aufsichtsrat und Vor- stand zu Karrierechancen von Frauen im Unternehmen zu stellen?} Ich bekam 2009 die üblichen Einladungen zu den Hauptversammlungen von Daimler und Munich Re, weil ich Aktien hatte. Im Internet habe ich mir angeschaut, wie Aufsichtsrat und Vorstand ausschauen und habe festgestellt, dass in den Vorständen keine und in den Aufsichtsräten so gut wie keine Frauen waren. Der Aufsichtsrat der Munich Re sollte insgesamt neu gewählt werden und zur Wahl standen nur Männer. Ich habe mir gesagt, so geht es nicht weiter. Wir müssen aktiv werden hinfahren und reden. Die Idee war nicht neu, ich hatte sie schon länger und hatte auch schon einmal mit Kolleginnen darüber in der Arbeitsgemeinschaft Anwältinnen gesprochen. Als ich diese Einladungen sah platzte mir der Kragen und ich dachte, jetzt ist es Zeit. Alle rieten zur Vorsicht, es sei so kompliziert, Anträge in einer Hauptversammlung seien schwierig. Aber ich wollte da nun hingehen. Eine Kollegin aus dem Vorstand des DAV, Margarete von Oppen, hat mich im Gesellschaftsrecht gecoacht und mitgeholfen, die Fragen zu formulieren, die wir bei Daimler gestellt haben. Das war der Beginn. Daraus habe ich ein Konzept entwickelt, dann ging es weiter und es wurde ein großartiges Projekt daraus. Jutta (Wagner, Präsidentin des djb von 2005-2011, Anm. Red.) war gleich begeistert von dieser Aktion und ist mit dem djb eingestiegen. Meine Idee war, man macht das bei mehreren Hauptversammlungen. Dass es ein solch umfangreiches Projekt geworden ist, hätte ich nicht gedacht. 
Wie hast Du Deine Rede auf Deiner ersten Hauptversammlung im April 2009 bei der Daimler AG in Berlin erlebt?

Ich erinnere mich gar nicht so richtig daran und weiß nur noch, dass ich es spannend fand. Ich habe die Fragen gestellt, die ich mir vorgenommen hatte, und sie kamen beim Podium auch an. Die Leute waren völlig platt, das hatten sie noch nie erlebt, aber der Vorstandsvorsitzende, Dieter Zetsche, ging auf die Fragen ein und bot ein Gespräch an. Man merkte, dass er aus den USA kam und etwas smoother war als die deutschen Haudegen. Das hat mir Mut gegeben, weiterzumachen. Ich war dann jedes Jahr bei mindestens zwei Hauptversammlungen, u.a. bei ThyssenKrupp, E.ON und Demag Cranes. Mit Petra Heinicke, der Vorsitzenden des Münchener Anwaltvereins, war ich auch bei BMW. Es hat Spaß gemacht. Aber vier Jahre sind nun auch genug.

\section{Wo nimmst Du die ganze Energie für Deine zahlreichen Aktivi- täten her?}

Meine Eltern sagten immer, du bist die älteste, du hast die meiste Kraft von uns mitbekommen. Irgendwie ist es schon die Natur, die einen mit dieser Energie gesegnet hat. Stressig war es, das kann man nicht leugnen, aber ich würde kein anderes Leben führen wollen. Gott sei Dank habe ich soviel Energie. Das hat vielleicht nicht jeder. Vor allem rege ich mich aber auch darüber auf, dass die Frauen immer noch in zweiter Reihe stehen und an die gläserne Decke stoßen. Das kann man sich einfach nicht gefallen lassen.
Warst Du schon immer ein bisschen aufsässig und unangepasst? Ich war schon immer so. Ich war die erste, die auf unserer Mädchenschule mit Lewis-Jeans ankam, das war völlig unüblich. Als junge Anwältin trug ich Miniröcke. Meine Mandanten, die Bauern haben gesagt, je kürzer der Rock, desto länger die Rechnung. Die Rechnung wurde immer bezahlt. Frauen haben die Chance sich abzuheben von den Anzugmännern. Ich habe die Chance genutzt und mich nicht in einen Business-Anzug gestürzt. Ich kann ein buntes Kleid oder enge Jeans anziehen und High Heels tragen und hatte nie das Bedürfnis, mich zu verstecken. Ich habe die Chance genutzt aufzufallen und mich abzuheben von diesem Einerlei der blau-schwarzen Anzüge. Ich weiß nicht, warum junge Kolleginnen heutzutage meinen, sie müssten sich in schwarz kleiden. Das zeugt für mich von mangelndem Mut. Aber der ist nicht jedem gegeben.

Als junge Studentin und Anwältin habe ich die Diskriminierung von Frauen nicht so bemerkt. Das geht jungen Frauen heutzutage auch so. Sie denken, die Welt steht einem offen und es kann einem nichts passieren. Man lernt erst später, dass es eine gläserne Decke gibt. Die hat mich zunehmend wütend gemacht und ich wollte etwas unternehmen. Jetzt finde ich es schön mit dem Juristinnenbund und den tollen Frauen zusammenarbeiten. Ich finde den djb sehr wichtig.

\section{Vielen Dank für das Gespräch!}

\section{Impressum}

Schriftleitung

Anke Gimbal, Rechtsassessorin (V.i.S.d.P.)

\section{Redaktionsanschrift}

Deutscher Juristinnenbund e. V.

Anklamer Str. 38

10115 Berlin

Telefon: $030443270-0$

Telefax: 030 443270-22

E-Mail: geschaeftsstelle@djb.de

www.djb.de

Erscheinungsweise:

4 Ausgaben im Jahr

\section{Bezugspreise 2014}

Jahresabonnement 54,- $€$; Einzelheft 15,- $€$. Alle Preise verstehen sich incl. MWSt, zzgl. Vertriebskostenanteil.

\section{Bestellmöglichkeit}

Bestellungen beim örtlichen Buchhandel oder direkt bei der Nomos Verlagsgesellschaft Baden-Baden

\section{Kündigungsfrist}

jeweils drei Monate vor Kalenderjahresende
Bankverbindung generell

Zahlungen jeweils im Voraus an Nomos Verlagsgesellschaft, Postbank Karlsruhe: IBAN: DE07 6601007500736367 51, BIC: PBNKDEFF oder Sparkasse Baden-Baden Gaggenau: IBAN: DE05 6625003000050022 66, BIC: SOLADES1BAD

\section{Druck und Verlag}

Nomos Verlagsgesellschaft $\mathrm{mbH} \& \mathrm{Co}$. KG Waldseestr. 3-5, D-76530 Baden-Baden Telefon (07221) 2104-O/Fax (07221) 2104-27 E-Mailnomos@nomos.de

\section{Anzeigen}

Sales friendly Verlagsdienstleistungen

Pfaffenweg 15, 53227 Bonn

Telefon (0228) 978980, Fax (o228) 9789820

E-Mail roos@sales-friendly.de

\section{Urheber- und Verlagsrechte}

Die Zeitschrift sowie alle in ihr enthaltenen einzelnen Beiträge und Abbildungen sind urheberrechtlich geschützt. Jede Verwertung, die nicht ausdrücklich vom Urheberrechtsgesetz zugelassen ist, bedarf der vorherigen Zustimmung des Verlags.

Mit der Annahme zur Veröffentlichung überträgt der Autor dem Verlag das ausschließliche Verlagsrecht für die Zeit bis zum Ablauf des Ur- heberrechts. Eingeschlossen sind insbesondere auch das Recht zur Herstellung elektronischer Versionen und zur Einspeicherung in Datenbanken sowie das Recht zu deren Vervielfältigung und Verbreitung online oder offline ohne zusätzliche Vergütung. Nach Ablauf eines Jahres kann der Autor anderen Verlagen eine einfache Abdruckgenehmigung erteilen; das Recht an der elektronischen Version verbleibt beim Verlag.

Namentlich gekennzeichnete Beiträge geben nicht in jedem Fall die Meinung der Herausgeber/Redaktion oder des Verlages wieder. Unverlangt eingesendete Manuskripte - für die keine Haftung übernommen wird - gelten als Veröffentlichungsvorschlag zu den Bedingungen des Verlages.

Die Redaktion behält sich eine längere Prüfungsfrist vor. Eine Haftung bei Beschädigung oder Verlust wird nicht übernommen. Bei unverlangt zugesandten Rezensionsstücken keine Garantie für Besprechung oder Rückgabe. Es werden nur unveröffentlichte Originalarbeiten angenommen. Die Verfasser erklären sich mit einer nicht sinnentstellenden redaktionellen Bearbeitung einverstanden.

ISSN 1866-377X 